\title{
Ruth M. Lynden-Bell, Simon Conway Morris, John D. Barrow, John L. Finney and Charles L. Harper Jr (Eds): Water and Life: The Unique Properties of $\mathrm{H}_{2} \mathrm{O}$
}

\author{
Ken Jones
}

Published online: 24 March 2011

(C) Springer-Verlag 2011

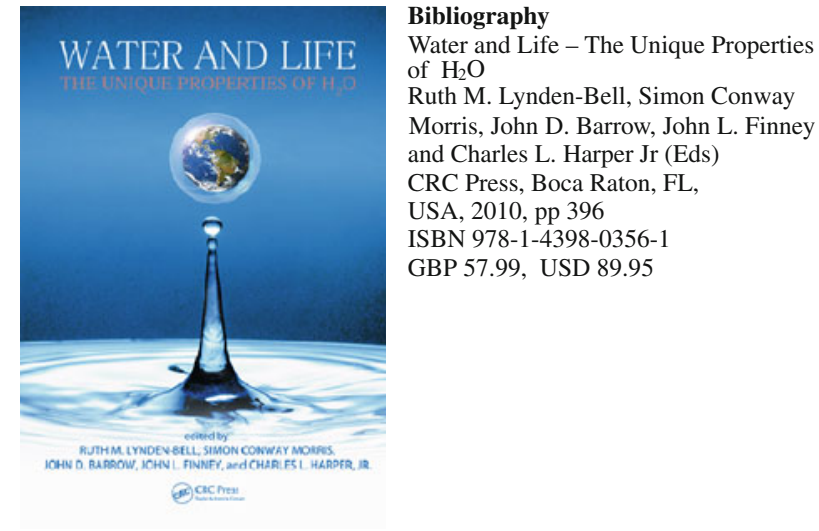

One view of nature's physical constants is that they are extraordinarily finely tuned to allow the existence of intelligent life; another is that evolution has capitalised on what was available. An attempt to resolve the issue led to the "Fitness of the Cosmos for Life" symposium, attended by biochemists, physicists, cosmologists and theologians. The cosmologists pointed out that a small alteration in the ratio of the kinetic energy at the time of the Big Bang would have totally changed the Universe; physicists played intellectual games; biochemists, with their vast unexplained chemical world, did not encourage any tinkering with physical constants; and theologians simply wanted to discuss design and teleology. This exposed an unbridgeable cultural gap between the various disciplines, but what did emerge led to a much narrower and less divisive subjectthe unique properties of water, convened as a second symposium, in 2005. This book is a collection of selected papers from this conference, specifically directed towards

K. Jones $(\bowtie)$

Knutsford, Cheshire, UK the structure, properties and interactions of water within the context of supporting life.

The primary key to life on Earth is water, in effect the universal solvent, with an ability to dissolve $\mathrm{CO}_{2}$, which is ionised and eventually deposited as limestone. Without this property, Earth would now be like Venus. Of primary interest to biochemists is how the apparently unremarkable water molecule plays totally unexpected roles and acts as a biomolecule in its own right. It provides a central role in both cellular mechanisms and macroscopic properties; when proteins fold, both hydrophobic and hydrophilic interactions are evident; there are exchanges of entropy; and there is remarkable latitude in the employment of hydrogen bonds. These effects are also echoed in polypeptide structures, dynamism on DNA surfaces and binder sites on ligands.

These extraordinary and wide ranging anomalous properties can only be unravelled by a study of the deeper levels of quantum mechanics and application of the Schrödinger equation, making explanations in the form of mathematical solutions. Even with these insights the "biofriendliness" of water still remains mysterious. That we still have much to learn becomes even more obvious as the knowledge imparted in this collection is absorbed, despite it adding a larger degree of understanding to anyone interested are fundamental questions; why, can, and how does life exist in the Universe?

This most intellectual collection of essays brings into focus how remarkable this ubiquitous molecule water really is. While defining much of what is known it also leaves many questions that still require answers, such as can the evolutionary process both cosmic and organic be regarded as biocentric? Any thinking scientist interested in the life processes will find this a totally fascinating read. 